\title{
On the reciprocal interaction between believing and feeling: an adaptive agent modelling perspective
}

\author{
Zulfiqar A. Memon $\cdot$ Jan Treur
}

Received: 27 February 2010/Revised: 30 August 2010/Accepted: 1 September 2010/Published online: 6 October 2010

(C) The Author(s) 2010. This article is published with open access at Springerlink.com

\begin{abstract}
An agent's beliefs usually depend on informational or cognitive factors such as observation or received communication or reasoning, but also affective factors may play a role. In this paper, by adopting neurological theories on the role of emotions and feelings, an agent model is introduced incorporating the interaction between cognitive and affective factors in believing. The model describes how the strength of a belief may not only depend on information obtained, but also on the emotional responses on the belief. For feeling emotions a recursive body loop between preparations for emotional responses and feelings is assumed. The model introduces a second feedback loop for the interaction between feeling and belief. The strength of a belief and of the feeling both result from the converging dynamic pattern modelled by the combination of the two loops. For some specific cases it is described, for example, how for certain personal characteristics an optimistic world
\end{abstract}

Parts of this article are based on work presented at the First International Conference on Brain Informatics, BI'09 (Memon and Treur 2009), and the Seventh International Symposium on Neural Networks, ISNN'10 (Memon and Treur 2010).

Z. A. Memon · J. Treur $(\square)$

Department of Artificial Intelligence, VU University

Amsterdam, De Boelelaan 1081, 1081 HV Amsterdam,

Netherlands

e-mail: treur@few.vu.nl

URL: http://www.few.vu.nl/ treur

Z. A. Memon

e-mail: zamemon@few.vu.nl

URL: http://www.few.vu.nl/ zamemon

Z. A. Memon

Sukkur Institute of Business Administration (Sukkur IBA),

Air Port Road Sukkur, Sindh, Pakistan view is generated in the agent's beliefs, or, for other characteristics, a pessimistic world view. Moreover, the paper shows how such affective effects on beliefs can emerge and become stronger over time due to experiences obtained. It is shown how based on Hebbian learning a connection from feeling to belief can develop. As these connections affect the strenghts of future beliefs, in this way an effect of judgment 'by experience built up in the past' or 'by gut feeling' can be obtained. Some example simulation results and a mathematical analysis of the equilibria are presented.

Keywords Believing $\cdot$ Feeling $\cdot$ Hebbian learning $\cdot$ Agent model

\section{Introduction}

When by observation or communication information about the world is acquired, it is often not completely clear and still subject to interpretation what has to be believed. For example, if from a distance you see a small paper attached at the front window of your car, you may start to believe that you received a charge for parking at the wrong place or time. Or you may start to believe that it concerns an advertising of a special offer. In such cases emotional aspects may become decisive in what you will believe. Such a process is the focus of the work reported here.

Already during the process that they are generated beliefs trigger emotional responses that result in certain feelings. However, the process of generation of a belief is not fully independent of such associated feelings. In a reciprocal manner, the generated feelings may also have a strengthening or weakening effect on the belief during this process. For example, by Frijda et al. (2000b) it is stated: 
According to appraisal theory, emotions result from how the individual believes the world to be, how events are believed to have come about, and what implications events are believed to have. Beliefs thus are regarded as one of major determinants of emotion, and therefore an important part of the study of emotion can properly be seen as falling under the umbrella of cognitive psychology. Oddly enough, however, the reverse direction of influence in the relation between emotion and cognition has received scant attention. This is in itself rather odd, because one midgt easily regard emotions as being among the determinants of an individual's beliefs, and their resistance to modification. Indeed, such an influence has traditionally been considered to be one of the most important things to be said about emotions. Spinoza (1677/1989) defined emotions as "states that make the mind inclined to think one thing rather than another". The influence of emotions upon beliefs can be vieweed as the port through which emotions exert their influence upon human life. (...)

The general proposal thus is that emotions can awaken, intrude into, and shape beliefs, by creating them, by amplifying or altering them, and by making them resistant to change.

(Frijda et al. 2000b, pp. 1, 5)

Recent empirical work such as described in, for example, Eich et al. (2000), Forgas et al. (2005, 2009), Niedenthal (2007), Schooler and Eich (2000), Winkielman et al. (2009), reports such types of effects of emotions on beliefs in experimental contexts (but does not relate them to neurological findings or theories).

The area of cognitive neuroscience is developing rapidly, thereby exploring possibilities for dynamic modelling approaches based on neurological knowledge (e.g., Purves et al. 2008; Gazzaniga 2009; Atmanspacher and Rotter 2008; Majumdar 2007; Dolan 2002; Frijda et al. 2000a; LaBar and Cabeza 2006; Pessoa 2008; Phelps 2006; Storbeck and Clore 2007). In this paper, adopting neurological theories on emotion and feeling, a computational dynamic agent model is introduced that models this reciprocal interaction between feeling and believing. The computational model, which is based on neurological theories on the embodiement of emotions as described, for example, in Damasio (1994, 1996, 1999, 2003) and Winkielman et al. (2009), describes how the generation of a belief may not only depend on an (external) informational source, but also takes into account how the belief triggers an emotional response that leads to a certain feeling. More specifically, in accordance with, for example Damasio (1999, 2003), for feeling the emotion associated to a belief a converging recursive body loop is assumed. A second converging feedback loop introduced in the model, inspired by the Somatic Marker Hypothesis (Damasio 1994, 1996), involves the interaction back from the feeling to the belief. Thus, a combination of two loops is obtained, where connection strengths within these loops in principle are person-specific. Depending on these personal characteristics, from a dynamic interaction within and between the two loops, an equilibrium is reached for both the strength of the belief and of the feeling.

Connections from feelings to beliefs are person-dependent and in fact constitute part of a person's personality. To illustrate the model, in the explanation of the model and simulations results reported, the following example scenario is used.

A person is parking his car for a short time at a place where this is not allowed. When he comes back, from some distance he observes that a small paper is attached at the front window of the car. He starts to generate the belief that the paper represents a charge to be paid. This belief generates a negative feeling, which, depending on the type of personality, for this case has an impact on the belief by strengthening it. Coming closer, some contours of the type of paper that is attached become visible. As these are not clearly recognized as often occurring for a charge, the person starts to generate a second belief, namely that it concerns an advertising of a special offer. This belief generates a positive feeling which, again depending on the type of personality, in this case has an impact on the latter belief by strengthening it.

Note that in this case the assumed personality of the person makes that both beliefs associated to negative feelings and beliefs associated to positive feelings are amplified. In the model such specific personality aspects easily can be changed by changing the values of the parameters in the model.

Personality aspects, as represented by certain parameters in the model, might be assumed given a priori (innate), but it can be questioned how plausible such an assumption is. A main focus in this paper is also on how the effect of the feeling on the belief can emerge over time based on personal experiences: it is shown how such connections in an agent model may automatically emerge by a Hebbian learning mechanism (cf. Hebb 1949; Bi and Poo 2001; Gerstner and Kistler 2002; Xie and Seung 2003). To model this, it is assumed that the connection from feeling to belief initally has strength zero, but due to a Hebbian learning mechanism over time gets nonzero strength. This means that the agent learns to strengthen its belief based on a supporting feeling. As a consequence, when such a feeling would be absent, the agent's belief would develop less strength; the feeling gives it its full strength. This principle models the idea that during lifetime a person builds up certain intuitions or gut feelings, and lets these play an important role in what to (fully) believe and what not to 
believe. More specifically, over time events occur in a person's life triggering a certain belief, and for each of these events the person experiences a certain feeling, which by Hebbian learning strengthens the association between feeling and belief. As a result in future situations when such a belief is triggered, the associated feeling is generated with a certain strength, which can be interpreted as 'having a gut feeling', or having a 'judgement by experience' for this belief, where the latter word 'experience' may be taken to refer to an accumulation of experienced feelings over the past events.

In this paper, first in "From believing to feeling and vice versa" Damasio's theory on the generation of feelings based on a body loop is briefly introduced. Moreover, the second loop is introduced, the one between feeling and belief. In "The computational model for believing and feeling" the model is described in detail. "Example simulation results of the interaction of belief and feeling" presents some simulation results. In "Learning to believe by feeling", the Hebbian learning model is described, and "Example simulation results for learning to believe" presents some simulation results of the resulting adaptive process. In "Mathematical analysis" a mathematical analysis of the equilibria of the model is presented. Finally, "Discussion" is a discussion.

\section{From believing to feeling and vice versa}

In this section the interaction between believing and feeling is discussed in some more detail from a neurological perspective, in both directions: from believing to feeling, and from feeling to believing. The notions of emotion and feeling, and their relationship are adopted from (Damasio 1999).

\section{From believing to feeling}

Damasio $(1999,2003)$ describes how in a person a belief state induces emotions felt within this person, as described by Damasio (1999, 2003); for example:

Even when we somewhat misuse the notion of feeling - as in "I feel I am right about this" or "I feel I cannot agree with you"-we are referring, at least vaguely, to the feeling that accompanies the idea of believing a certain fact or endorsing a certain view. This is because believing and endorsing cause a certain emotion to happen. As far as I can fathom, few if any exceptions of any object or event, actually present or recalled from memory, are ever neutral in emotional terms. Through either innate design or by learning, we react to most, perhaps all, objects with emotions, however weak, and subsequent feelings, however feeble. (Damasio 2003, p. 93)
Notice that in Damasio (1999) a distinction is made between an emotion (or emotional response), which is considered a preparation for a bodily response triggered by some cause, and a feeling for this emotion, which is generated by forming a sensory representation for the body state induced as an emotional response. Damasio (1999) explains emotion as follows:

The substrate for the representation of emotions is a collection of neural dispositions in a number of brain regions (...) They exist, rather, as potential patterns of activity arising within neuron ensembles. Once these dispositions are activated, a number of consequences ensue. On the one hand, the pattern of activation represents, within the brain, a particular emotion as 'neural object'. On the other, the pattern generates explicit responses that modify both the state of the body proper and the state of other brain regions. By so doing, the responses create an emotional state, and at that point, an external observer can appreciate the emotional engagement of the organism being observed. (Damasio 1999, p. 79)

Here the substrate for the representation of an (internal) emotional state is considered a collection of neural dispositions in the brain, which are activated as a reaction on a certain stimulus. Once this occurs, it entails modification of both the body state (which can be considered as an expressed, externally observable emotional state), and the state of other brain regions. By these events, an emotional state is created which is accessible for external observation; this state may have multiple facets or dimensions. In schematic form, emotion generation and feeling the emotion via a body loop roughly proceeds according to the following causal chain; see Damasio (1999, 2003):

belief $\rightarrow$ preparation for the induced bodily response

$\rightarrow$ body modification $\rightarrow$ sensing the body state $\rightarrow$

sensory representation of the body state $\rightarrow$ feeling

As a variation, an 'as if body loop' uses a direct causal relation

preparation for the induced bodily response $\rightarrow$ sensory representation of the body state

as a shortcut in the causal chain. The body loop (or as if body loop) is extended to a recursive body loop (or recursive as if body loop) by assuming that the preparation of the bodily response is also affected by the state of feeling the emotion:

feeling $\rightarrow$ preparation for the bodily response

as an additional causal relation. Such recursiveness is also assumed by Damasio (2003), as he notices that what is felt 
by sensing is actually a body state which is an internal object, under control of the person:

The brain has a direct means to respond to the object as feelings unfold because the object at the origin is inside the body, rather than external to it. The brain can act directly on the very object it is perceiving. It can do so by modifying the state of the object, or by altering the transmission of signals from it. The object at the origin on the one hand, and the brain map of that object on the other, can influence each other in a sort of reverberative process that is not to be found, for example, in the perception of an external object. (...)

In other words, feelings are not a passive perception or a flash in time, especially not in the case of feelings of joy and sorrow. For a while after an occasion of such feelings begins - for seconds or for minutes there is a dynamic engagement of the body, almost certainly in a repeated fashion, and a subsequent dynamic variation of the perception. We perceive a series of transitions. We sense an interplay, a give and take. (Damasio 2003, pp. 91-92)

Thus the obtained model is based on reciprocal causation relations between emotion felt and body states, as roughly shown in Fig. 1.

Within the model presented in this paper both the bodily response and the feeling are assigned a level or gradation, expressed by a number, which is assumed dynamic; for example, the strength of a smile and the extent of happiness. The causal cycle is modelled as a positive feedback loop, triggered by a belief and converging to a certain level of feeling and body state. Here in each round of the cycle the next body state has a level that is affected by both the belief and the level of the feeling state, and the next level of the feeling is based on the level of the body state.

From feeling to believing

In an idealised rational agent the generation of beliefs might only depend on informational sources and be fully independent from non-informational aspects such as emotions. However, in real life persons may, for example, have

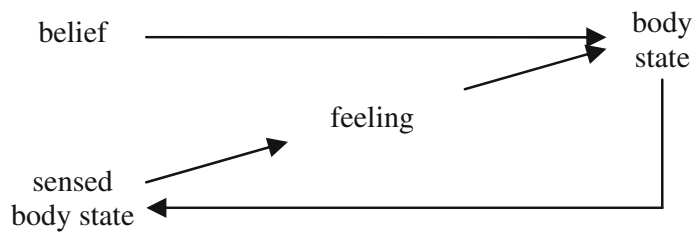

Fig. 1 Body loop induced by a belief a more optimistic or pessimistic character and affect their beliefs in the sense that an optimist person strengthens beliefs that have a positive feeling associated and a pessimistic person strengthens beliefs with a negative associated feeling. Thus the strengths of beliefs may depend on noninformational aspects of mental processes and related personal characteristics. To model this for the case of feelings a causal relation

$$
\text { feeling } \rightarrow \text { belief }
$$

can be added. This introduces a second recursive loop, as shown in Fig. 2.

Given a causal path (by a body loop or as-if body loop) making a connection from belief to feeling, from a neurological perspective the existence of a connection backward from feeling to belief may be considered plausible, as due to the forward connection, neurons involved in the belief and in the associated feeling will often be activated simultaneously: whenever a certain belief is activated, the induced emotional response will activate the feeling as well. Therefore such a connection from feeling to belief may be developed based on a general Hebbian learning mechanism (Hebb 1949; Bi and Poo 2001) that strengthens connections between neurons that are activated simultaneously, similar to what has been proposed for the emergence of mirror neurons; e.g., Keysers and Perrett (2004) and Keysers and Gazzola (2009). The mechanism by which this takes place will be modelled in "Learning to believe by feeling".

Another type of support for a connection from feeling to belief can be found in Damasio's Somatic Marker Hypothesis (cf. Damasio 1994, 1996; Bechara and Damasio 2005; Damasio 2003). This is a theory on decision making which provides a central role to emotions felt. Each decision option induces (via an emotional response) a feeling which is used to mark the option. For example, when a negative somatic marker is linked to a particular option, it provides a negative feeling for that option. Similarly, a positive somatic marker provides a positive feeling for that option. Damasio describes the use of somatic markers in the following way:

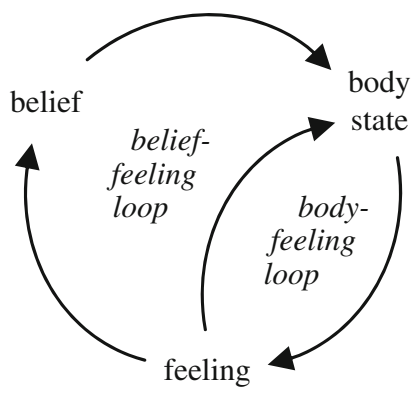

Fig. 2 The two recursive loops related to a belief 
the somatic marker (..) forces attention on the negative outcome to which a given action may lead, and functions as an automated alarm signal which says: Beware of danger ahead if you choose the option which leads to this outcome. The signal may lead you to reject, immediately, the negative course of action and thus make you choose among other alternatives. (...) When a positive somatic marker is juxtaposed instead, it becomes a beacon of incentive. (...) on occasion somatic markers may operate covertly (without coming to consciousness) and may utilize an 'as-if-loop'. (Damasio 1994, pp. 173-174)

Usually the Somatic Marker Hypothesis is applied to provide endorsements or valuations for options for a person's actions. However, it may be considered plausible that such a mechanism is applicable to valuations of internal states such as beliefs as well.

\section{The computational model for believing and feeling}

Informally described theories or models in scientific disciplines, for example, in biological or neurological contexts, often are formulated in terms of causal relationships (e.g., Seth 2008), or dynamical relationships between states as described in dynamical systems (e.g., Port and van Gelder 1995). In particular, this applies to what was discussed in "From believing to feeling and vice versa". To obtain a manageable explanation or model, it is convenient to choose an appropriate abstraction level to express such causal or dynamical relationships. Establishing these relationships in neurological context requires that timing aspects of temporal associations between different neural activations are to be taken into account (e.g., Wilmer et al. 2010). To adequately formalise such theories or models the hybrid dynamic modelling language LEADSTO has been developed that subsumes qualitative and quantitative causal modelling approaches, and dynamical systems modelling approaches (cf. Bosse et al. 2007a). This language has been proven successful in a number of contexts, varying from biochemical processes that make up the dynamics of cell behaviour (cf. Jonker et al. 2008) to neurological and cognitive processes (e.g., Bosse et al. 2007b, c, 2008). Within LEADSTO the temporal relation

$\mathrm{a} \rightarrow \mathrm{b}$

denotes that whenever a state property a occurs (for example, the fact that the activation level of a certain neuron or group of neurons has a certain value), then after a certain time delay, state property $b$ (for example, the fact that the activation level of another neuron or group of neurons has a certain value) will occur. This time delay (for example, the step size $\Delta t$ in discrete forms of differential equations) can be specified for each relation instance as any positive real number. In the hybrid language LEADSTO both logical and numerical calculations can be specified in an integrated manner, and a dedicated software environment is available to support specification and simulation.

An overview of the agent model for believing and feeling is depicted in Fig. 3. The detailed specifications of the agent model are presented below. The picture also shows representations from the detailed specifications. However, note that the precise numerical relations between the indicated variables $\mathrm{V}$ shown are not expressed in the picture in Fig. 3, but in the detailed specifications of properties below. Here capitals are used for variables, and lower case letters for specific instances. To support reproducibility and reusability of the model in the hybrid causal modelling style used in the work reported in this paper (and for those more accustomed to this type of format), in Boxes 1 and 2, the detailed formal specifications in LEADSTO format are included, labeled by LP1 to LP9 as also shown in the picture.

First the part is presented that describes the basic mechanisms to generate a belief state and the associated emotional response. The first dynamic property addresses how properties of the world state can be sensed. Note that the model enables the use of different world state properties at the same time, which can be sensed and processed further. Therefore for world state properties the variable $\mathrm{W}$ is used which can be used to range over specific instances. For the sake of simplicity it is assumed that the world state properties $\mathrm{W}$ that are modelled, can be observed.

\section{LP1 Sensing a world state}

If world state property $\mathrm{W}$ occurs of strength $V$ then a sensor state for $\mathrm{W}$ of strength $V$ will occur

For the example scenario this dynamic property is used by the agent to observe both the paper attached looking like a charge and the paper type looking like an offer; to this end the variable $\mathrm{W}$ is instantiated by specific instances charge and offer. Note that to indicate that not only $\mathrm{V}$ is a variable, but also $\mathrm{W}$, the formal notation world_state $(W, V)$ is used in Figs. 1 and 2 and in Boxes 1 and 2 ; this expression indicates that world state property $\mathrm{W}$ has activation value or strenght $\mathrm{V}$. This notation also could be written (with the same meaning) alternatively as world_state (W) (V) or world_statew $(V)$. The same applies to other expressions involving world states $\mathrm{W}$ or body states B. In Figs. 2 and 3 small letters w and b are used to indicate specific instances.

From the sensor states, sensory representations are generated according to the dynamic property LP2. Note that also here it is specified as a general rule, for which in 
Fig. 3 A model for the dynamics of believing and feeling based on a body loop

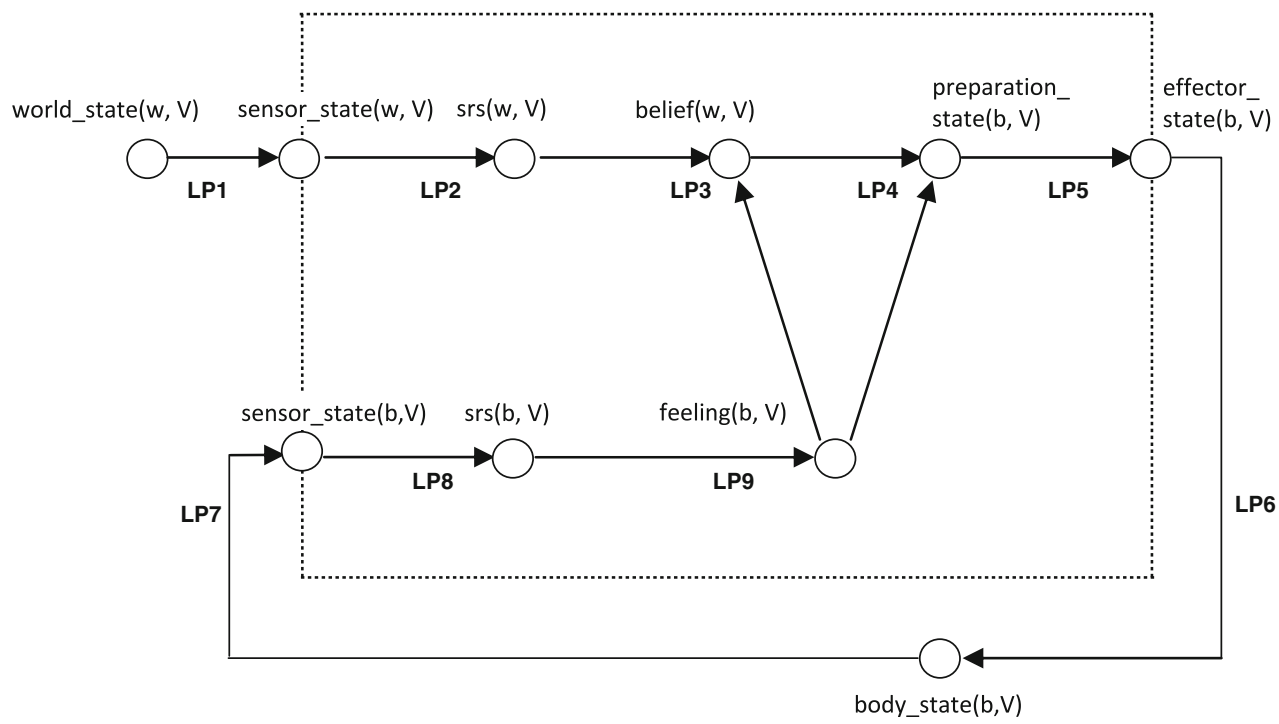

the example the variable $\mathrm{W}$ is instantiated as indicated above.

\section{LP2 Generating a sensory representation for a sensed world state}

If a sensor state for world state property $\mathrm{W}$ with level $V$ occurs,

then a sensory representation for $\mathrm{W}$ with level $V$ will occur

Next the property is described that relates strengths of a specific sensory representation of an instance $w$ and a specific feeling of an instance $b$ to the strength of the specific belief on $w$, thereby using certain strengths of the connections involved. Here a connection strength $\omega_{1}$ from sensory representation to belief and $\omega_{2}$ from feeling to belief is assumed. So, note that this property will not hold universally for all instances of world states $W$ and body states $B$.

In "Example simulation results of the interaction of belief and feeling" it will be discussed how the connection strength $\omega_{2}$ is adapted by a Hebbian learning principle. A function $g\left(\beta_{1}, \omega_{1}, \omega_{2}, V_{1}, V_{2}\right)$ is used for the way in which activation levels $V_{1}$ and $V_{2}$ of sensory representation and feeling are combined taking into account the connection strengths. Here $\beta_{1}$ is a parameter indicating the person's orientation for believing; value 0 indicates that the person is reluctant to believe and 1 that the person is willing to amplify the strength of his or her belief, whereas a value of 0.5 makes that the person takes the average values of the two sources. Moreover, $\gamma_{1}$ is a parameter indicating the person's speed or flexibility in change of a belief; here value 0 indicates that the person will never change a belief, and 1 that the person is immediately willing to change the belief.

\section{LP3 Generating a belief state for a feeling and a sensory representation}

If a sensory representation for w with strength $V_{1}$ occurs, and the associated feeling of $\mathrm{b}$ has strength $V_{2}$

and the belief for w has strength $V_{3}$

and the connection from sensory representation to belief of w has strength $\omega_{1}$

and the connection from feeling $\mathrm{b}$ to belief of $\mathrm{w}$ has strength $\omega_{2}$

and $\quad \beta_{1}$ is the person's orientation for believing

and $\gamma_{1}$ is the person's flexibility for beliefs

then after $\Delta t$ the belief for $\mathrm{w}$ will have strength $V_{3}+\gamma_{1}\left(g\left(\beta_{1}, \omega_{1}, \omega_{2}, V_{1}, V_{2}\right)-V_{3}\right) \Delta t$

Note that this property can be written in differential equation format as follows:

$d V_{3} / d t=\gamma_{1}\left(g\left(\beta_{1}, \omega_{1}, \omega_{2}, V_{1}, V_{2}\right)-V_{3}\right)$

with variable names for quantities as indicated in the antecedent of property LP3 above. The resulting level for the belief is calculated based on a function $g\left(\beta_{1}, \omega_{1}, \omega_{2}, V_{1}\right.$, $\left.V_{2}\right)$ of the original levels. For the function $g\left(\beta_{1}, \omega_{1}, \omega_{2}, V_{1}\right.$, $V_{2}$ ) the following was taken:

$$
\begin{aligned}
g\left(\beta_{1}, \omega_{1}, \omega_{2}, V_{1}, V_{2}\right)= & \beta_{1}\left(1-\left(1-\omega_{1} V_{1}\right)\left(1-\omega_{2} V_{2}\right)\right) \\
& +\left(1-\beta_{1}\right) \omega_{1} \omega_{2} V_{1} V_{2}
\end{aligned}
$$

Note that this formula describes a weighted sum of two cases. The most positive case considers the two source values as strengthening each other, thereby staying under 1 : combining the imperfection rates (i.e., their deviation from the value 1) $1-V_{1}$ and $1-V_{2}$ of them provides a decreased rate of imperfection expressed by $1-\left(1-V_{1}\right)\left(1-V_{2}\right)$. The most negative case considers the two source values in a negative combination: combining the imperfections of 
Box 1 LEADSTO

specifications for the model, part I: generating a belief and preparation

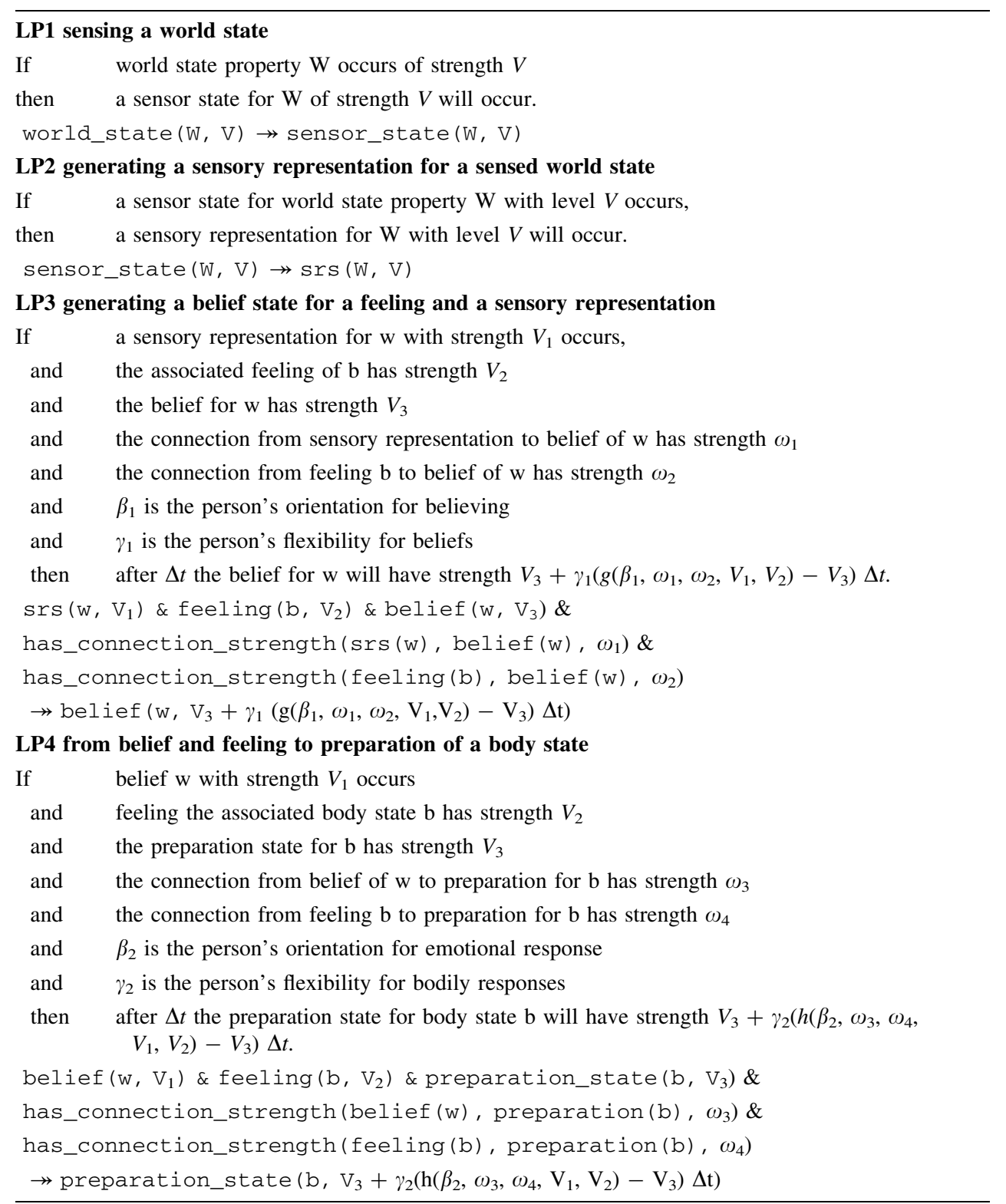

them provides an increased imperfection; this is expressed by $V_{1} V_{2}$. This function $g\left(\beta_{1}, \omega_{1}, \omega_{2}, V_{1}, V_{2}\right)$ can be considered to play the role of a quadratic threshold function, parameterised by $\beta_{1}$. Note that for connection strength $\omega_{2}=0$ (no effect of feeling on belief) the formula reduces to the following:

$g\left(\beta_{1}, \omega_{1}, 0, V_{1}, V_{2}\right)=\beta_{1}\left(1-\left(1-\omega_{1} V_{1}\right)\right)=\beta_{1} \omega_{1} V_{1}$

In the example simulations discussed in "Example simulation results of the interaction of belief and feeling" the connection strength $\omega_{1}$ has been set on 1 .

Note that the function $g$ in property LP3 (and also $h$ in property LP4 below) has been kept abstract, so that they can easily be filled with specific functions different from the functions used here. As an example, it would be quite well possible to use, for example, a discrete threshold function

$$
\begin{aligned}
g\left(\tau, \omega_{1}, \omega_{2}, V_{1}, V_{2}\right)= & 1 \text { if } \omega_{1} V_{1} \\
& +\omega_{2} V_{2} \geq \tau, \text { and } 0 \text { otherwise }
\end{aligned}
$$

with $\tau$ a threshold parameter, or a continuous logistic threshold function

$g\left(\sigma, \tau, \omega_{1}, \omega_{2}, V_{1}, V_{2}\right)=1 /\left(1+e^{-\sigma\left(\omega_{1} V_{1}+\omega_{2} V_{2}-\tau\right)}\right)$

with $\sigma$ a steepness parameter and $\tau$ a threshold parameter.

Dynamic property LP4 describes the (internal) emotional state (see "From believing to feeling") as a response to a specific belief on an instance $w$; this emotional state has the form of the preparation for a specific bodily 
Box 2 LEADSTO

specifications for the model, part II: body loop and as-if body loop

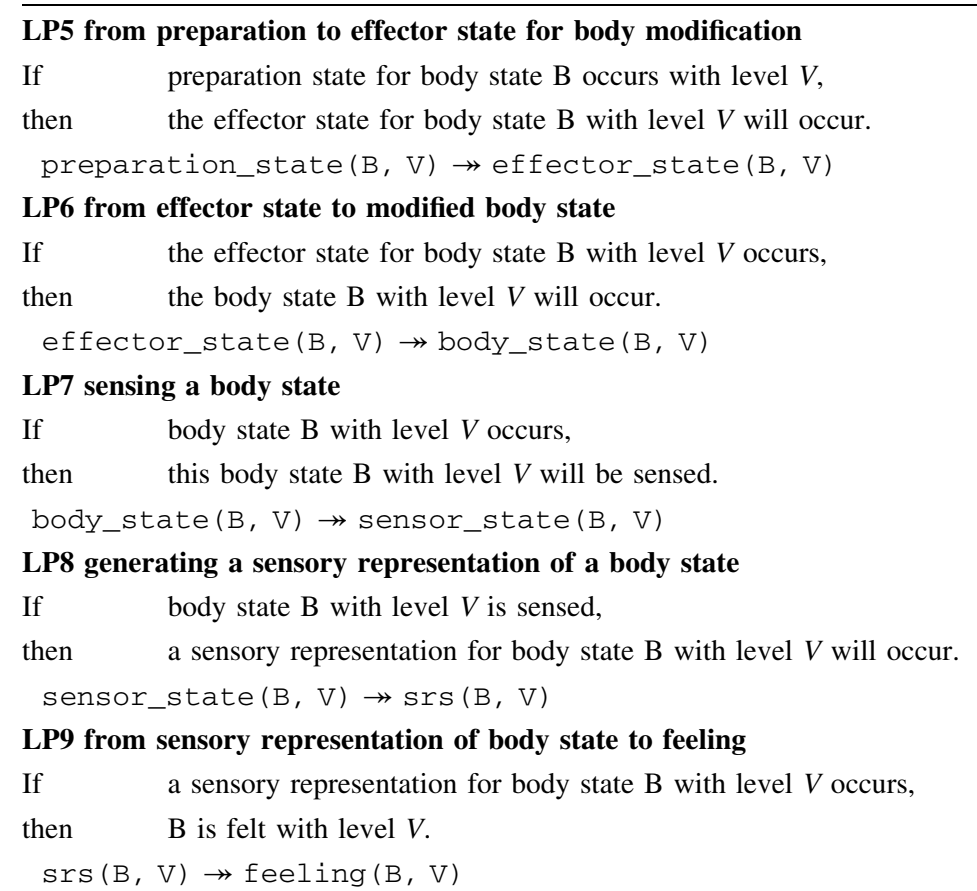

LP10 From preparation to sensory representation of a body state

If preparation state for body state B occurs with level $V$,

then a sensory representation for body state B with level $V$ will occur.

preparation_state $(B, V) \rightarrow \operatorname{srs}(B, V)$ reaction instance $b$. Note that the actual (modified) body state can be considered as an expressed, externally observable emotional state. Also this property does not hold for all instances $w$ and $b$. The resulting level for the preparation is calculated based on a function $h\left(\beta_{2}, \omega_{3}, \omega_{4}, V_{1}, V_{2}\right)$ of the original levels. Here $\omega_{3}$ is the connection strength from belief to preparation and $\omega_{4}$ from feeling to preparation. Moreover $\beta_{2}$ is a parameter indicating the person's orientation for preparing responses; value 0 indicates that the person is reluctant to respond and 1 that the person is willing to amplify the strength of his or her response, and a value of 0.5 makes that the person takes the average values of the two sources. Moreover, $\gamma_{2}$ is a parameter indicating the person's speed or flexibility in change of a preparation; here value 0 indicates that the person will never change a preparation, and 1 that the person is immediately willing to change the preparation.

\section{LP4 From belief and feeling to preparation of a body state}

If belief w with strength $V_{1}$ occurs

and feeling the associated body state $\mathrm{b}$ has strength $V_{2}$

and the preparation state for $\mathrm{b}$ has strength $V_{3}$

and the connection from belief of $w$ to preparation for $b$ has strength $\omega_{3}$ and the connection from feeling $\mathrm{b}$ to preparation for $\mathrm{b}$ has strength $\omega_{4}$

and $\beta_{2}$ is the person's orientation for emotional response

and $\quad \gamma_{2}$ is the person's flexibility for bodily responses

then after $\Delta t$ the preparation state for body state $\mathrm{b}$ will have strength $V_{3}+\gamma_{2}\left(h\left(\beta_{2}, \omega_{3}, \omega_{4}, V_{1}, V_{2}\right)-V_{3}\right)$ $\Delta t$

Note that this property can be written in differential equation format as follows:

$d V_{3} / d t=\gamma_{2}\left(h\left(\beta_{2}, \omega_{3}, \omega_{4}, V_{1}, V_{2)}-V_{3}\right)\right.$

with variable names for quantities as indicated in the antecedent of property LP4 above. For the function $h\left(\beta_{2}\right.$, $\left.\omega_{3}, \omega_{4}, V_{1}, V_{2}\right)$ the following has been taken:

$$
\begin{aligned}
h\left(\beta_{2}, \omega_{3}, \omega_{4}, V_{1}, V_{2}\right)= & \beta_{2}\left(1-\left(1-\omega_{3} V_{1}\right)\left(1-\omega_{4} V_{2}\right)\right) \\
& +\left(1-\beta_{2}\right) \omega_{3} \omega_{4} \mathrm{~V}_{1} \mathrm{~V}_{2}
\end{aligned}
$$

In the example simulations discussed in "Example simulation results of the interaction of belief and feeling" the connection strengths $\omega_{3}$ and $\omega_{4}$ have been set on 1 .

Dynamic properties LP5 to LP9 describe the body loop. Like LP1 and LP2 they hold for all instances for variables $B$ and $W$. 
LP5 From preparation to effector state for body modification

If preparation state for body state B occurs with level $V$,

then the effector state for body state B with level $V$ will occur

\section{LP6 From effector state to modified body state}

If the effector state for body state $B$ with level $V$ occurs,

then the body state B with level $V$ will occur

\section{LP7 Sensing a body state}

If body state B with level $V$ occurs,

then this body state B with level $V$ will be sensed

LP8 Generating a sensory representation of a body state

If body state $\mathrm{B}$ with level $V$ is sensed,

then a sensory representation for body state B with level $V$ will occur

\section{LP9 From sensory representation of body state to feeling}

If a sensory representation for body state B with level $V$ occurs,

then B is felt with level $V$

Alternatively, dynamic properties LP5 to LP8 describing the body loop can also be replaced by one dynamic property LP10 describing an as-if body loop as follows; see Fig. 4.

\section{LP10 From preparation to sensory representation of a body state}

If preparation state for body state B occurs with level $V$, then the effector state for body state B with level $V$ will occur
Example simulation results of the interaction of belief and feeling

Based on the model described in the previous section, a number of simulations have been performed. Some example simulation traces are included in this section as an illustration; see Figs. 5 and 6 (here the time delays within the temporal LEADSTO relations were taken 1 time unit). In Fig. 5 two different traces are shown with different personal characteristics as expressed by the specific values of the parameters of the model. Note that the scaling of the vertical axis differs per graph. For both traces the world state shows the development of the strength of the belief that the observed paper (observed with a rather modest strength of 0.3 ) at the car is an offer. Moreover both $\gamma_{1}=0.6$ and $\gamma_{2}=0.6$. Simulation trace 1 at the left hand side has $\beta_{1}=0.5$ and $\beta_{2}=0.5$, whereas simulation trace 2 at the right hand side has $\beta_{1}=0.5$ and $\beta_{2}=1$. Note that if no specific personality is kept in mind, such choices of parameter values are to a certain extent arbitrary. For example, the person modelled in trace 2 depicted in Fig. 5 (with $\beta_{2}=1$ ) has as a personal characteristic a stronger tendency to let the feeling positively affect the strength of the belief than the person modelled in trace 1 (with $\beta_{2}=0.5$ ), as can be seen in the figure.

In trace 1 the belief (and also the feeling) gets the same strength as the stimulus, namely 0.3 ; here no effect of the emotional response is observed. However, in trace 2 the belief gets a higher strength (namely 0.65) due to the stronger emotional response (with feeling getting strength 1). This shows how a belief can be affected in a substantial manner by the feedback from the emotional response on the belief.

In Fig. 6 the complete example scenario for the car parking case discussed earlier is shown. Note that in the upper part of this figure (and in Figs. 7, 8, 9), at the vertical
Fig. 4 A model for the dynamics of believing and feeling based on an as-if body loop

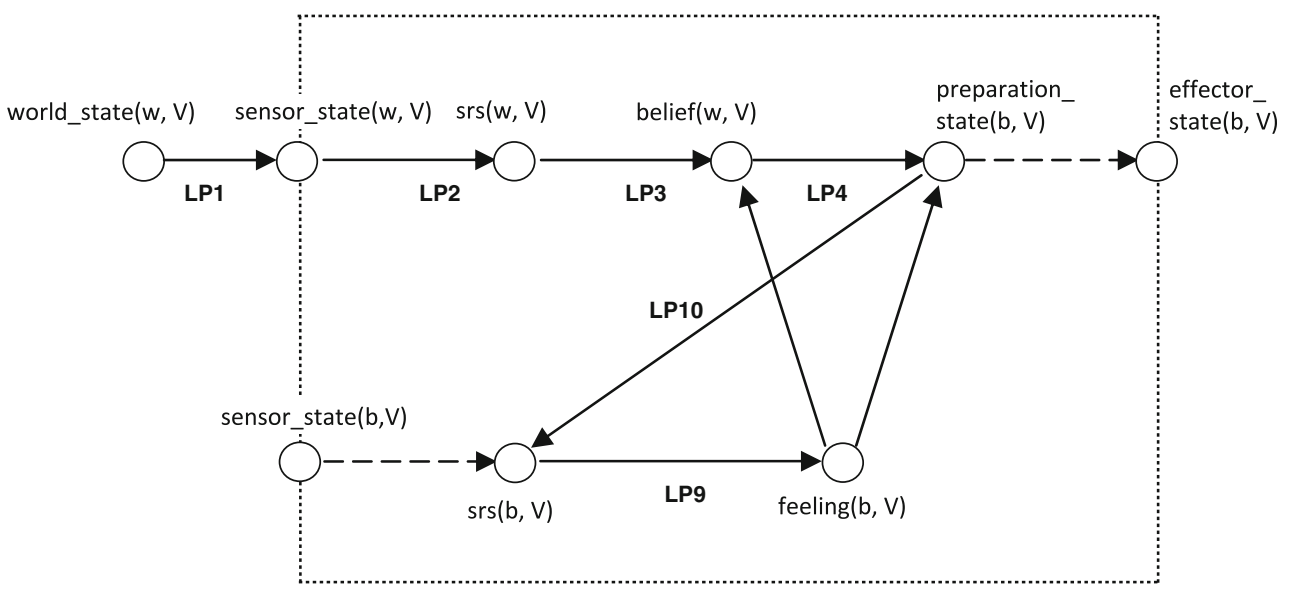



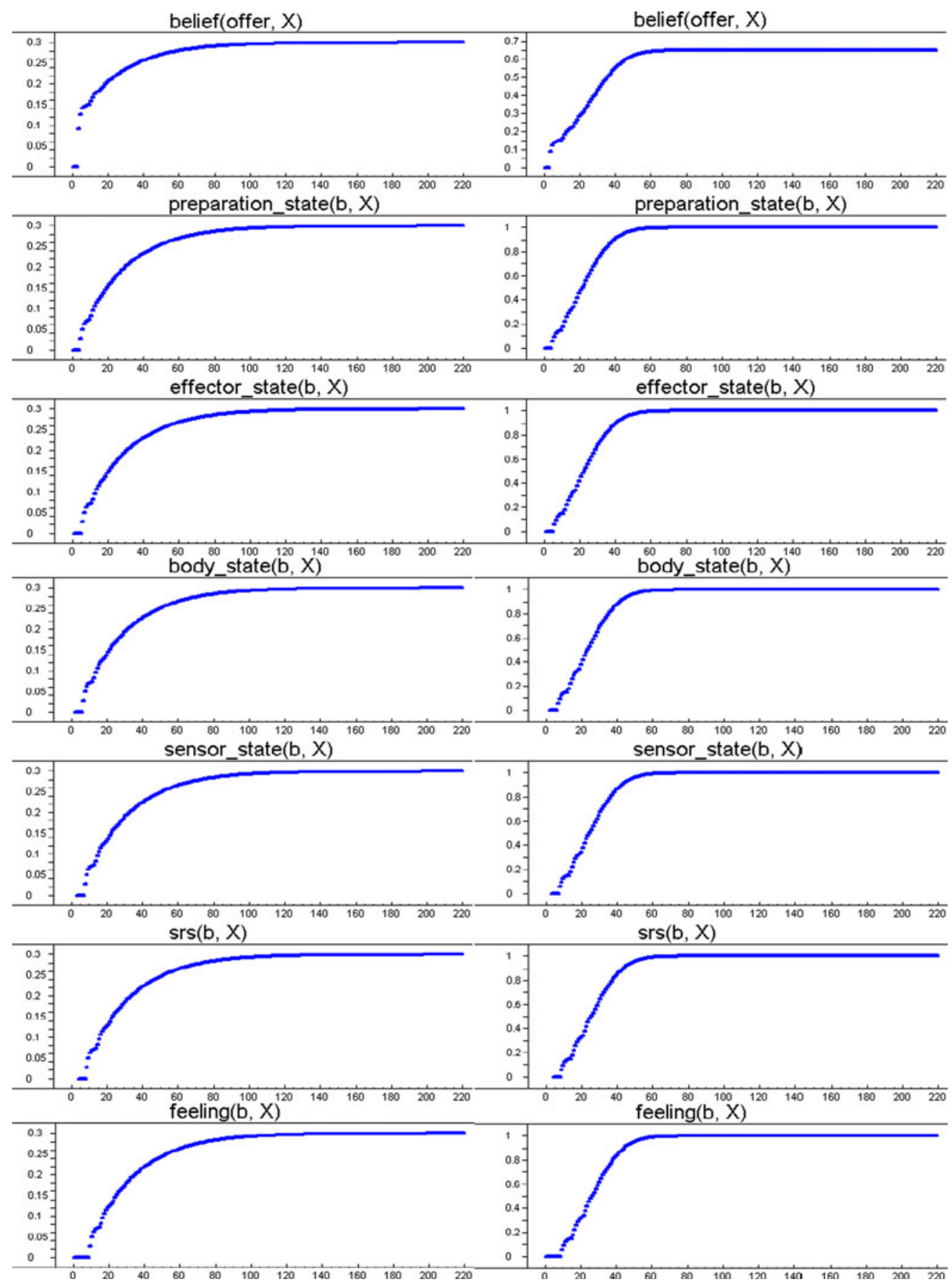

Fig. 5 Two example traces: (1) $\beta_{1}=0.5$ and $\beta_{2}=0.5$, (2) $\beta_{1}=0.5$ and $\beta_{2}=1$

axis state properties are represented which can hold or not at specific time points (represented at the horizontal axis). A dark line indicates a time interval for which a state property shown at the vertical axis holds, and a light line an interval for which it does not hold. The world state shows something that (from a distance) looks like a charge with strength 0.8 until time point 225; this is indicated by the fifth dark line (for state property world_state(charge, 0.8)) in the upper part of Fig. 6. For this case $\beta_{1}=0.8$ and $\beta_{2}=0.4$ was taken, which means a modest role for the emotional response. The belief in a charge leads to an increasingly strong emotional body state $b 1$ and via the related feeling, the belief reaches a strength a bit above 0.9 .

However, having come closer to the car, after time point 225 the world state shows with strength 0.8 something that is more like an offer, whereas the strength of the charge 


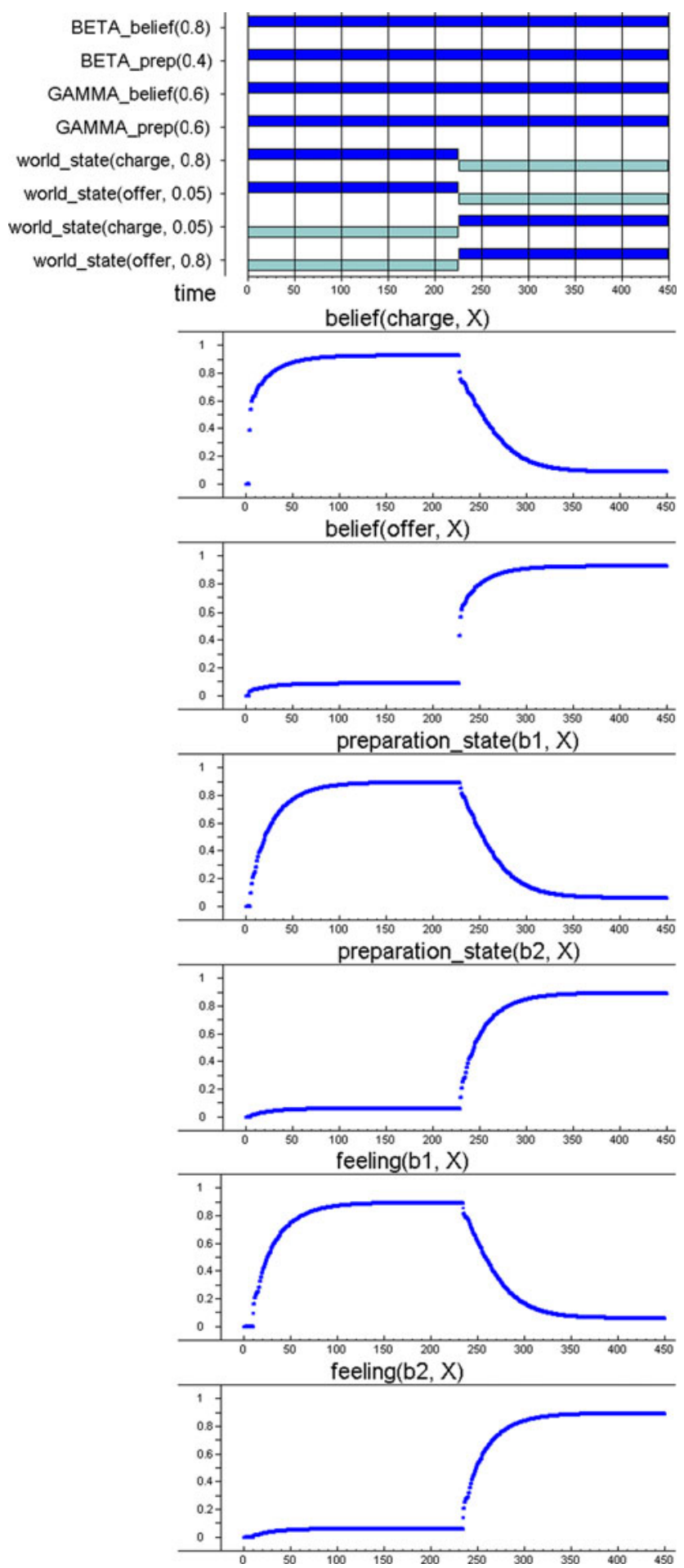

Fig. 6 Trace for the car parking case with $\beta_{1}=0.8$ and $\beta_{2}=0.4$

shown drops to 0.05 , which also was the strength of the offer before time point 225 . As a consequence the belief in a charge drops and based on a different emotion response on the offer belief based on body state $b 2$ the strength of the belief in an offer increases until above 0.9.

\section{Learning to believe by feeling}

In "From believing to feeling and vice versa" the connection from feeling to belief was motivated by Damasio's neurological theory called the Somatic Marker Hypothesis. One of the elements of this theory is that somatic markers depend on past experiences of the person. Within the agent model introduced above this element is incorporated by making the connection strength from feeling to believing adaptive, dependent on beliefs and feelings experienced over time. From a Hebbian neurological perspective (Hebb 1949), strengthening of a connection from feeling to belief over time may be considered plausible, as neurons involved in the belief and in the associated feeling will often be activated simultaneously. Therefore such a connection from feeling to belief may be developed and adapted based on a Hebbian learning mechanism (Hebb 1949; Bi and Poo 2001; Gerstner and Kistler 2002): connections between neurons that are activated simultaneously are strengthened, similar to what has been proposed for the emergence of mirror neurons in, e.g., Keysers and Perrett (2004), Keysers and Gazzola (2009), see also Diwadkar et al. (2008).

Based on these considerations, in the agent model the connection strength $\omega$ is adapted using the following Hebbian learning rule. It takes into account a maximal connection strength 1 , a learning rate $\eta$, and an extinction rate $\zeta$. A similar Hebbian learning rule can be found in Gerstner and Kistler (2002, p. 406).

By the factor $(1-\omega)$ the learning rule keeps the level of $\omega$ bounded by 1 (which could be replaced by any number), as Hebbian learning without such a bound usually provides instability. When extinction is neglected, the upward changes during learning are proportional to both $V_{1}$ and $V_{2}$, which in particular means that no learning takes place whenever one of them is 0 , and maximal learning takes place when both are 1 .

\section{LP11 Hebbian learning rule}

If the feeling of $\mathrm{b}$ has strength $V_{1}$

and the belief for $w$ has strength $V_{2}$

and the connection from feeling $b$ to belief of $w$ has strength $\omega$

and the learning rate from feeling $\mathrm{b}$ to belief of $\mathrm{w}$ is $\eta$ and the extinction rate from feeling $\mathrm{b}$ to belief of $\mathrm{w}$ is $\zeta$ then after $\Delta t$ the connection from feeling $\mathrm{b}$ to belief of $\mathrm{w}$ will have strength $\omega+\left(\eta V_{1} V_{2}(1-\omega)-\zeta \omega\right) \Delta t$

feeling $\left(b, V_{1}\right)$ \& belief $\left(w, V_{2}\right)$ \& has_connection_strength $(b, w, \omega) \&$ has_learning_rate $(b, w, \eta) \&$ has_ extinction_rate $(b, w, \zeta)$

$\rightarrow$ has_connection_strength(b, w, $\omega+$ $\left.\left(\eta \mathrm{V}_{1} \mathrm{~V}_{2}(1-\omega)-\zeta \omega\right) \Delta t\right)$ 


\section{Example simulation results for learning to believe}

Based on the agent model described in the previous section, a number of simulations have been performed. Some example simulation traces are included in this section as an illustration; see Figs. 7, 8 and 9 (the time delays within the LEADSTO relations were taken 1 time unit). Here the connection strengths $\omega_{1}, \omega_{3}$ and $\omega_{4}$ have been set on 1 , and $\omega_{2}$ is dynamic based on the Hebbian learning rule.

In Fig. 7, $\beta_{1}=0.95, \beta_{2}=0.4, \eta=0.02$ and the extinction rate $\zeta$ is 0.0002 . The example trace in Fig. 7 shows how after learning of the connection from feeling to belief, the strength of the belief substantially exceeds the strength of the incoming stimulus (0.9 vs. 0.6).
Fig. 7 Example trace with a number of learning phases

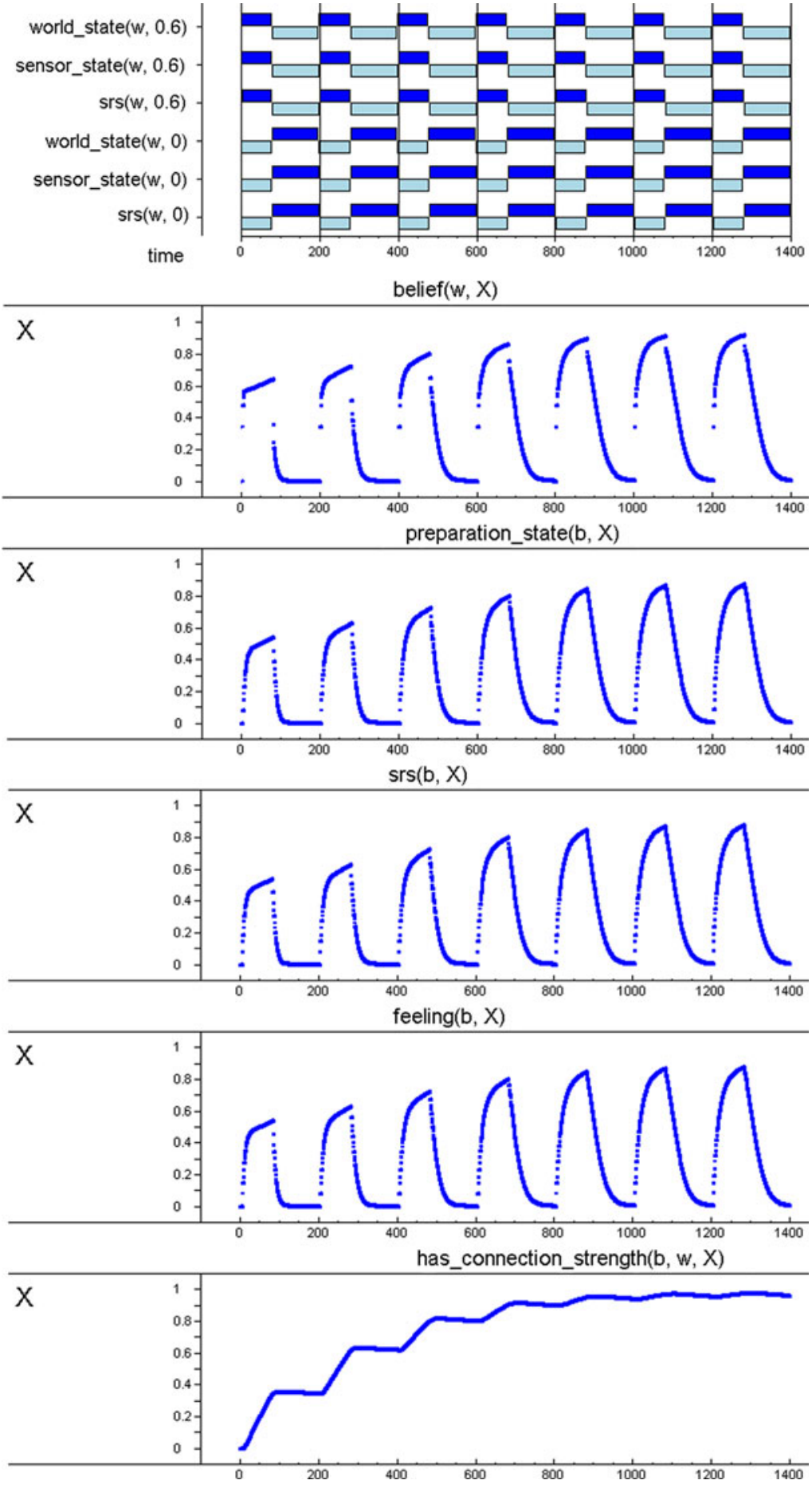




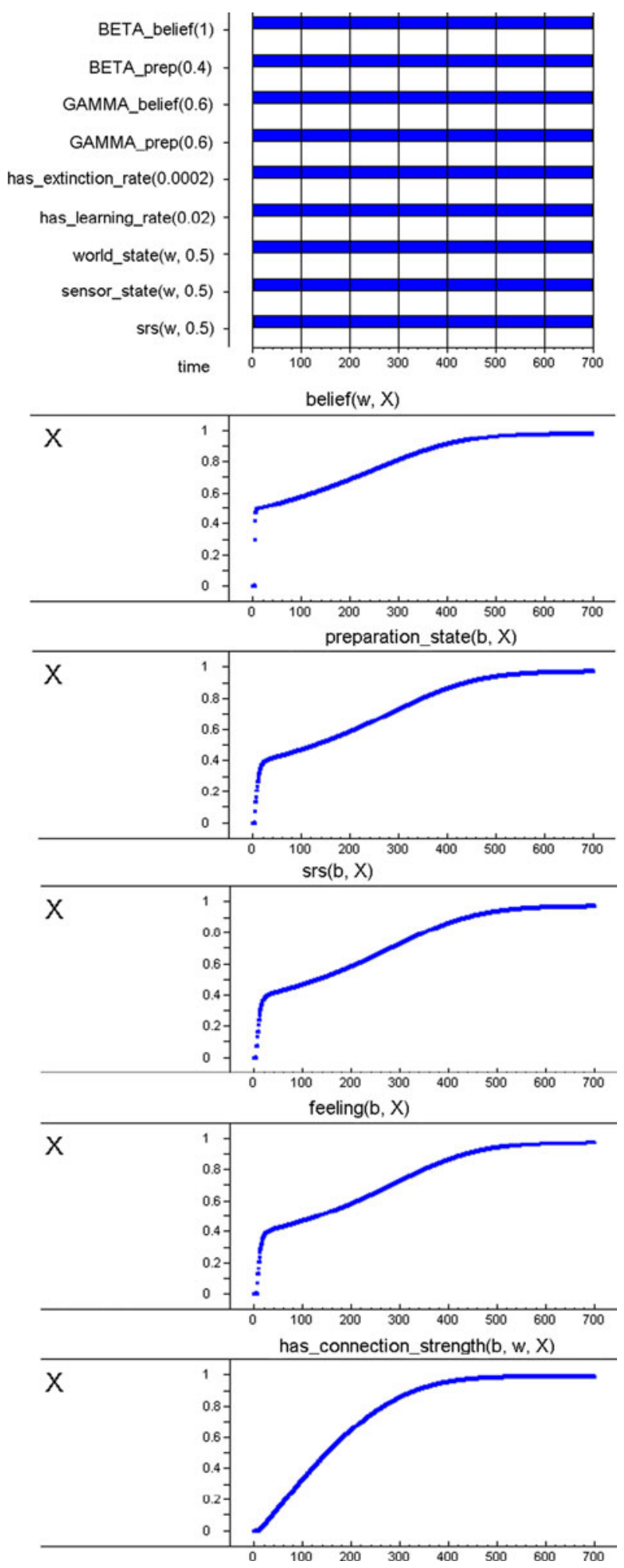

Fig. 8 Approximated equilibrium for $\beta_{1}=1, \beta_{2}=0.4$

In Figs. 8 and 9 some example simulation traces are showing how equilibria are reached for a constant environment with settings as indicated in the upper part of the figures. Note that, in contrast to the situation in Fig. 7

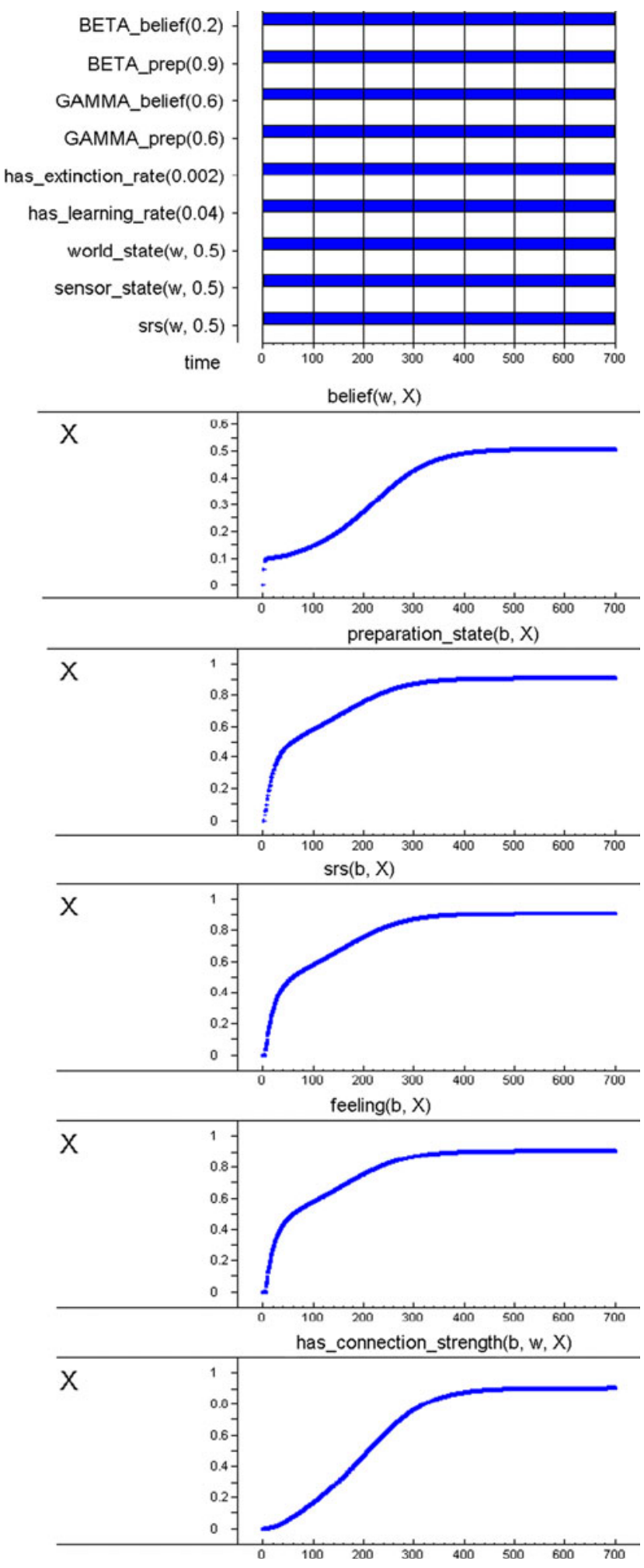

Fig. 9 Approximated equilibrium for $\beta_{1}=0.2, \beta_{2}=0.9$

where the world is fluctuating in providing information, in Figs. 8 and 9 the same information from the world state is constantly coming in, forever. Given this stable environmental factor, all variables of the model stabelise as well: 
they all will approximate their equilibrium value. The equilibrium values reached in these traces illustrate the outcomes of the mathematical analysis of equilibria that will be presented in "Mathematical analysis".

\section{Mathematical analysis}

In the example simulations discussed in "Example simulation results of the interaction of belief and feeling" it was shown that for a time period with a constant environment, the strengths of beliefs, body states and feelings reach a stable equilibrium. By a mathematical analysis it can be addressed which types of equilibria are possible. To this end equations for equilibria can be determined from the dynamical model equations for the belief and the preparation state level, which can be expressed as differential equations (with $b(t)$ the level of the belief, $s(t)$ of the stimulus, $f(t)$ of the feeling, and $p(t)$ of the preparation for the body state at time $t$ ).

\section{Non-adaptive case}

First, for the sake of simplicity, the case of a fixed connection strength 1 from feeling to belief is addressed. The following differential equations can be obtained.

$$
\begin{aligned}
d b(t) / d t= & \gamma_{1}\left(\beta_{1}(1-(1-s(t))(1-f(t)))\right. \\
& \left.+\left(1-\beta_{1}\right) s(t) f(t)-b(t)\right) \\
d p(t) / d t= & \gamma_{2}\left(\beta_{2}(1-(1-b(t))(1-f(t)))\right. \\
& \left.+\left(1-\beta_{2}\right) b(t) f(t)-p(t)\right)
\end{aligned}
$$

To obtain equations for equilibria, constant values for all variables are assumed (also the ones that are used as inputs such as the stimuli). Then in all of the equations the reference to time $t$ can be left out, and in addition the derivatives $d b(t) / d t$ and $d p(t) / d t$ can be replaced by 0 . Assuming $\gamma_{1}$ and $\gamma_{2}$ nonzero, this leads to the following equations. $\beta_{1}(1-(1-s)(1-f))+\left(1-\beta_{1}\right) s f-b=0$

$\beta_{2}(1-(1-b)(1-f))+\left(1-\beta_{2}\right) b f-p=0$

As for an equilibrium it also holds that $f=p$, this results in the following two equations in $b, f$, and $s$ :

$$
\begin{aligned}
& \beta_{1}(1-(1-s)(1-f))+\left(1-\beta_{1}\right) s f-b=0 \\
& \beta_{2}(1-(1-b)(1-f))+\left(1-\beta_{2}\right) b f-f=0
\end{aligned}
$$

For the general case (1) can directly be used to express $b$ in $f, s$ and $\beta_{1}$. Using this, in (2) $b$ can be replaced by this expression in $f, s$ and $\beta_{1}$, which transforms (2) into a quadratic equation in $f$ with coefficients in terms of $s$ and the parameters $\beta_{1}$ and $\beta_{2}$. Solving this quadratic equation algebraically provides a complex expression for $f$ in terms of $s, \beta_{1}$ and $\beta_{2}$. Using this, by (1) also an expression for $b$ in terms of $s, \beta_{1}$ and $\beta_{2}$ can be found. As these expressions become rather complex, only an overview for a number of special cases is shown in Table 1 (for nine combinations of values $0,0.5$ and 1 for both $\beta_{1}$ and $\beta_{2}$ ). For these cases the Eqs. 1 and 2 can be substantially simplified as shown in the second column (for Eq. 1) and second row (for Eq. 2).

As can be seen in this table, persons that are pessimistic for believing $\left(\beta_{1}=0\right)$ and have a negative profile in generating emotional responses $\left(\beta_{2}=0\right)$, reach a stable equilibrium for which both the belief and the feeling have level 0 . The opposite case occurs when a person is optimistic for believing $\left(\beta_{1}=1\right)$ and has a positive profile in generating emotional responses $\left(\beta_{2}=1\right)$. Such a person reaches a stable equilibrium for which both the belief and the feeling have level 1. For cases where one of these $\beta_{1}$ and $\beta_{2}$ is 0 and the other one is 1 , a stable equilibrium is reached where the belief gets the same level as the stimulus: $b=s$. When a person is in the middle between optimistic and pessimistic for believing $\left(\beta_{1}=0.5\right)$, for the case of a negative profile in generating emotional responses the stable belief reached gets half of the level of the stimulus, whereas for the case of a positive profile in generating

\begin{tabular}{|c|c|c|c|c|c|c|}
\hline & $\beta_{2}$ & 0 & & 0.5 & 1 & \\
\hline$\beta_{1}$ & & $f=0$ & $b=1$ & $b=f$ & $f=1$ & $b=0$ \\
\hline 0 & $b=s f$ & $b=f=0$ & $b=f=s=1$ & $\begin{array}{l}b=f=0 \\
\text { or } \\
b=f \text { and } s=1\end{array}$ & $\begin{array}{l}b=s \\
f=1\end{array}$ & $\begin{array}{l}b=s=0 \\
\text { or } \\
b=f=0\end{array}$ \\
\hline 0.5 & $b=(s+f) / 2$ & $\begin{array}{l}b=s / 2 \\
f=0\end{array}$ & $b=f=s=1$ & $b=f=s$ & $\begin{array}{l}b=(s+1) / 2 \\
f=1\end{array}$ & $b=f=s=0$ \\
\hline 1 & $\begin{array}{l}1-b=(1-s) \\
\quad(1-f)\end{array}$ & $\begin{array}{l}b=s \\
f=0\end{array}$ & $\begin{array}{l}b=f=1 \\
\text { or } \\
b=s=1\end{array}$ & $\begin{array}{l}b=f=1 \\
\text { or } \\
b=f \text { and } s=0\end{array}$ & $b=f=1$ & $b=f=s=0$ \\
\hline
\end{tabular}
emotional responses the stable belief reached gets 0.5 above half of the level of the stimulus (which is the 0.65
Table 1 Overview of equilibria for 9 non-adaptive cases of parameter settings 
shown in the second trace in Fig. 5). This clearly shows the effect of the feeling on the belief. The case where both $\beta_{1}=0.5$ and $\beta_{2}=0.5$ is illustrated in the first trace in Fig. 5: $b=f=s$.

\section{Adaptive case}

Next the adaptive case is addressed where the connection strength is not kept rigid, but is also changing during the process of reaching an equilibrium; as part of this process, it reaches its own equilibrium. The example simulations in Figs. 8 and 9 show how for a time period with a constant environment with strength 0.5 , the strengths of beliefs, body states and feelings and connection between feeling and belief reach a stable equilibrium. For this case the following differential equations can be obtained.

$$
\begin{aligned}
d b(t) / d t= & \gamma_{1}\left(\beta_{1}\left(1-\left(1-\omega_{1} s(t)\right)\left(1-\omega_{2}(t) f(t)\right)\right)\right. \\
& \left.+\left(1-\beta_{1}\right) \omega_{1} \omega_{2}(t) s(t) f(t)-b(t)\right) \\
d p(t) / d t= & \gamma_{2}\left(\beta_{2}\left(1-\left(1-\omega_{3} b(t)\right)\left(1-\omega_{4} f(t) f(t)\right)\right)\right. \\
& \left.+\left(1-\beta_{2}\right) \omega_{3} \omega_{4} b(t) f(t)-p(t)\right) \\
d \omega_{2}(t) / d t= & \left(\eta b(t) f(t)\left(1-\omega_{2}(t)\right)-\zeta \omega_{2}(t)\right)
\end{aligned}
$$

Note that below, as in "Example simulation results for learning to believe" the connection strengths $\omega_{1}, \omega_{3}$ and $\omega_{4}$ are taken 1. Moreover, $\omega_{2}$ is denoted as $\omega$. Assuming $\gamma_{1}, \gamma_{2}, \zeta$ and $\eta$ nonzero, this leads to the following equations.

$$
\begin{array}{r}
\beta_{1}(1-(1-s)(1-\omega f))+\left(1-\beta_{1}\right) \omega s f-b=0 \\
\beta_{2}(1-(1-b)(1-f))+\left(1-\beta_{2}\right) b f-p=0 \\
\eta b f(1-\omega)-\zeta \omega=0
\end{array}
$$

As for an equilibrium it also holds that $f=p$, this results in the following equations in $b, f, \omega, s$ :

$$
\begin{aligned}
& \beta_{1}(1-(1-s)(1-\omega f))+\left(1-\beta_{1}\right) \omega s f-b=0 \\
& \beta_{2}(1-(1-b)(1-f))+\left(1-\beta_{2}\right) b f-f=0 \\
& \eta b f(1-\omega)-\zeta \omega=0
\end{aligned}
$$

Note that as an extreme case $b=f=s=0$ satisfies (3), (4) and (5). For the general case, first, Eq. 5 can be rewritten into

$$
\begin{aligned}
\eta b f-\omega \eta b f-\zeta \omega & =0 \\
\omega(\eta b f+\zeta) & =\eta b f \\
\omega & =\frac{\eta b f}{\eta b f+\zeta} \\
\omega & =\frac{1}{1+\frac{\zeta}{\eta b f}}
\end{aligned}
$$

where the last step only applies when $b, f \neq 0$. Using $b$, $f \leq 1$ from this it follows that $\omega \leq \frac{1}{1+\frac{\zeta}{\eta}}<1$

This shows that given the extinction, the maximal connection strength will be lower than 1 , but may be close to 1 when the extinction rate is very small compared to the learning rate. However, it also depends on the equilibrium values for $f$ and $b$. For values of $f$ and $b$ that are 1 or close to 1 , this maximal value of $\omega$ can be approximated. When in contrast these values are low, also the equilibrium value for $\omega$ will be low, since:

$\omega=\frac{\eta b f}{\eta b f+\zeta} \leq \frac{\eta b f}{\zeta}$

In particular, when one of $b$ and $f$ is 0 then also $\omega$ is 0 (and conversely).

For the general case Eq. 3 can directly be used to express $b$ in $f, \omega, s$ and $\beta_{1}$. Using this, in (4) $b$ can be replaced by this expression in $f, \omega, s$ and $\beta_{1}$, which transforms (4) into a quadratic equation in $f$ with coefficients in terms of $s, \omega$ and the parameters $\beta_{1}$ and $\beta_{2}$. Solving this quadratic equation algebraically provides a complex expression for $f$ in terms of $s, \omega, \beta_{1}$ and $\beta_{2}$. Using this, by (3) also an expression for $b$ in terms of $s, \omega, \beta_{1}$ and $\beta_{2}$ can be found.

Again only an overview for a number of special cases is shown in Table 2. For these cases the Eqs. 3 and 4 can be substantially simplified as shown in the second column (for Eq. 3) and second row (for Eq. 4). As can be seen in Table 2, persons that have a low orientation for believing $\left(\beta_{1}=0\right)$ and have a low profile in generating emotional responses $\left(\beta_{2}=0\right)$, have an equilibrium for which both the belief and the feeling have level 0 , and also $\omega=0$. The case where both $\beta_{1}=0.5$ and $\beta_{2}=0.5$ indicates an equilibrium with $b=f=s$, and $\omega=1 /\left(1+\zeta / \eta s^{2}\right)$. Note that in Table 2 for $\beta_{1}=1$ and $\beta_{2}$ nonzero, two equations in $\omega$ and $b$ occur, which can be solved further to obtain more complex explicit expressions for each of them.

\section{Discussion}

In this paper, first an agent model was introduced incorporating the reciprocal interaction between believing and feeling based on neurological theories that address the role of emotions and feelings. A belief usually triggers an emotional response. Conversely, a belief may not only depend on information obtained, but also on this emotional response, as, for example, shown in literature such as Eich et al. (2000), Forgas et al. (2005, 2009), Niedenthal (2007), Schooler and Eich (2000). In the literature, this phenomenon has been studied informally but no formal computational models have been developed, as far as the authors 
Table 2 Overview of equilibria for nine adaptive cases of parameter settings for $\beta_{1}$ and $\beta_{2}$

\begin{tabular}{|c|c|c|c|c|c|c|}
\hline & $\beta_{2}$ & 0 & & 0.5 & 1 & \\
\hline$\beta_{1}$ & & $f=0$ & $b=1$ & $b=f$ & $f=1$ & $b=0$ \\
\hline 0 & $b=\omega s f$ & $b=f=\omega=0$ & - & $b=f=\omega=0$ & $\begin{array}{l}b=\omega s \\
f=1 \\
\omega=0 \text { or } \omega=1-\frac{\zeta}{\eta s}\end{array}$ & $b=\omega=0$ \\
\hline 0.5 & $b=(s+f) / 2$ & $\begin{array}{l}b=s / 2 \\
f=\omega=0\end{array}$ & $\begin{array}{l}b=f=s=1 \\
\omega=\frac{1}{1+\frac{5}{n}}\end{array}$ & $\begin{array}{l}b=f=s \\
\omega=\frac{1}{1+\frac{\zeta}{n s^{2}}}\end{array}$ & $\begin{array}{l}b=(s+1) / 2 \\
f=1 \\
\omega=\frac{1}{1+\frac{2 \zeta}{\eta(s+1)}}\end{array}$ & $b=f=s=\omega=0$ \\
\hline 1 & $1-b=(1-s)(1-\omega f)$ & $\begin{array}{l}b=s \\
f=\omega=0\end{array}$ & $\begin{array}{l}b=f=\omega=1 \\
\text { or } \\
b=s=1 \\
\omega=\frac{1}{1+\frac{\zeta}{\eta f}}\end{array}$ & $\begin{array}{l}b=f \\
\omega=\frac{1}{1+\frac{\zeta}{\eta b^{2}}} \\
\omega=\frac{1-\left(\frac{s}{b}\right)}{1-s}\end{array}$ & $\begin{array}{l}f=1 \\
\omega=\frac{b-s}{1-s} \\
\omega=\frac{1}{1+\frac{\zeta}{\eta b}}\end{array}$ & $\begin{array}{l}b=s= \\
\omega=0\end{array}$ \\
\hline
\end{tabular}

know. Accordingly, this paper reports an attempt to develop a formal computational model of how a belief generates an emotional response that is felt, and on the other hand how the emotion that is felt affects the belief. For feeling the emotion, based on elements taken from Damasio (1999, 2003), and Bosse et al. (2008), a converging recursive body loop is included in the model. As a second loop the model includes a converging feedback loop for the interaction between feeling and belief. Both the strength of the belief and of the feeling emerge as a result of the dynamic pattern generated by the combination of the two loops.

The causal relation from feeling to belief in this second loop was inspired by the Somatic Marker Hypothesis described in Damasio (1994, 1996), Bechara and Damasio (2005), and may also be justified by a Hebbian learning principle (cf. Hebb 1949; Bi and Poo 2001), as also has been done for the functioning of mirror neurons; e.g., Keysers and Perrett (2004), Keysers and Gazzola (2009). To formalise this adaptive process, a model for the connection from feeling to belief was developed based on a Hebbian learning rule (cf. Hebb 1949; Bi and Poo 2001; Gerstner and Kistler 2002; Xie and Seung 2003). The resulting adaptive model on the one hand describes how a belief generates an emotional response that is felt, and on the other hand how a connection can emerge enabling that the emotion that is felt affects the strength of the belief. The models were specified in the hybrid dynamic modelling language LEADSTO, and simulations were performed in its software environment (cf. Bosse et al. 2007a). A mathematical analysis of the equilibria of the models was discussed.

In the literature much support can be found for the idea that emotions or moods may affect beliefs. One example is the citation from Frijda et al. (2000b) in the introduction section. Other forms of support, also empirically, can be found in literature such as Forgas (1995, 2000), Clore and Gasper (2000), Clark and Brissette (2000), Eich et al. (2000), Forgas et al. (2005, 2009), Niedenthal (2007), Schooler and Eich (2000), Brun et al. (2008), Dolan (2002), Frijda et al. (2000a), LaBar and Cabeza (2006), Pessoa (2008), Phelps (2006), and Storbeck and Clore (2007). In principle the model is able to show patterns of effects of emotions on beliefs, which are qualitatively similar to patterns that have been reported for specific circumstances in literature, which can be viewed as a rough, qualitative kind of validation of the model.

However, in humans the effect of emotion or mood on belief is not always the same; a more differentiated point of view is put forward, for example, by Forgas (2000):

Numerous mundane everyday experiences are capable of inducing affect. (...) Such everyday moods often have a mood-congruent influence on many cognitive tasks (...) The cognitive consequences of moods are neither simple, nor straightforward, however. While numerous studies found a clear pattern of mood congruence in thoughts and judgements, many other experiments fail to find mood congruence, and even report an opposite, mood-incongruent effect on cognitions. (see Forgas 1995, for a detailed review) (Forgas 2000, p. 109)

Given such differentiations, a more specific validation of the model is a challenge on its own, involving a number of nontrivial aspects (including the issue of quantitative comparison: how strong is the effect on a belief), and aspects of subjective personal contextual situations and personal characteristics. For example, consider the situation that two persons $A$ and $B$ both are in contact with a third person $C$, and both desparately try to persuade her to have an exclusive relationship with them. Moreover, 
Table 3 Toy example showing how emotion-effects on beliefs depend on combinations of personality and contextual aspects

\begin{tabular}{lllllll}
\hline Information & A bias & B bias & A believes a & B believes a & A believes b & B believes b \\
\hline a & Positive & Positive & + & - & - & - \\
a & Positive & Negative & + & + & - & - \\
a & Negative & Positive & - & - & - & - \\
a & Negative & Negative & - & + & - & - \\
b & Positive & Positive & - & - & - & - \\
b & Positive & Negative & - & - & + \\
b & Negative & Positive & - & - & - \\
b & Negative & Negative & - & & + \\
\hline
\end{tabular}

consider information $a$ coming from a (not always reliable) friend of $C$, stating that $C$ intends to choose for $A$, compared to information $b$ that $C$ intends to choose for $B$, coming from the same person. Suppose for $A$ and $B$ 's personal characteristics as a biases for beliefs associated with positive resp. negative feelings are taken. Then, given the different subjective contexts, and depending on $A$ and $B$ 's personal characteristics, still all possible combinations of beliefs on $a$ or $b$ for $A$ and $B$ may occur, see Table 3. For example, if $A$ has a bias for negative feelings, he will not believe $a$, whereas in case $B$ has a bias for negative feelings, she will believe $a$. Only in case $A$ has a positive bias and $B$ a negative bias, both will believe $a$, and in the opposite case neither of them will believe $a$.

This toy example illustrates that a validation of any model as a general mechanism for the interaction between belief and feeling is far from trivial. Whether or not a feeling will have effect on a belief depends on a combination of different types of factors, among which personality aspects and subjective contextual aspects (in particular, including what the fact to be believed means for the person). Any experimental setup for validation has to deal with these combinatorics of individual and contextual differences. As this is a challenge on its own, a detailed validation of the model has not been conducted yet. Such a validation will be addressed as part of planned future work. One of the specific challenges here is to provide estimated values for the parameters representing individual personality characteristics (for example, the parameters $\beta_{1}$ and $\beta_{2}$, which represent a form of positive or negative orientation, and $\gamma_{1}$ and $\gamma_{2}$, which represent the speed or flexibility by which beliefs are changed). Experience with this issue for other models has shown the feasibility of an automated (on the fly) parameter tuning approach; for example, see Bosse et al. (2009), and Both et al. (2009). Furthermore, as a next step it will be explored how the presented model can be integrated within a software agent model in order to obtain a human-aware ambient agent that is able to estimate how a person's beliefs are affected both by information received and emotional responses.
Open Access This article is distributed under the terms of the Creative Commons Attribution Noncommercial License which permits any noncommercial use, distribution, and reproduction in any medium, provided the original author(s) and source are credited.

\section{References}

Atmanspacher H, Rotter S (2008) Interpreting neurodynamics: concepts and facts. Cogn Neurodyn 2:297-318

Bechara A, Damasio A (2005) The somatic marker hypothesis: a neural theory of economic decision. Games Econ Behav $52: 336-372$

Bi GQ, Poo MM (2001) Synaptic modifications by correlated activity: Hebb's postulate revisited. Ann Rev Neurosci 24:139-166

Bosse T, Jonker CM, Meij L, van der Treur J (2007a) A language and environment for analysis of dynamics by simulation. Int $\mathbf{J}$ Artif Intell Tools 16:435-464

Bosse T, Jonker CM, Treur J (2007b) Simulation and analysis of adaptive agents: an integrative modelling approach. Adv Complex Syst J 10:335-357

Bosse T, Jonker CM, Los SA, Torre L, van der Treur J (2007c) Formal analysis of trace conditioning. Cogn Systems Res J 8:36-47

Bosse T, Jonker CM, Treur J (2008) Formalisation of Damasio's theory of emotion feeling and core consciousness. Conscious Cogn J 17:94-113

Bosse T, Memon ZA, Treur J, Umair M (2009) An adaptive humanaware software agent supporting attention-demanding tasks. In: Proceedings of the 12th international conference on principles of practice in multi-agent systems, PRIMA'09. Lecture Notes in Artificial Intelligence. Springer Verlag, Berlin, 2009, pp 292-307

Both F, Hoogendoorn M, Jaffry SW, Lambalgen R van, Oorburg R, Sharpanskykh A, Treur J, de Vos M (2009) Adaptation and validation of an agent model of functional state and performance for individuals. In: Proceedings of the 12th international conference on principles of practice in multi-agent systems, PRIMA'09. Lecture Notes in Artificial Intelligence. Springer Verlag, Berlin, 2009, pp 595-607

Brun G, Doguoglu U, Kuenzle D (eds) (2008) Epistemology and emotions. Ashgate, Aldershot

Clark MS, Brissette I (2000) Relationship beliefs and emotion: reciprocal effects. In: Frijda NH et al (eds) Emotions and beliefs: how feelings influence thoughts. Cambridge University Press, Cambridge, pp 212-240

Clore GL, Gasper K (2000) Feeling is believing: some affective influences on belief. In: Frijda N, Manstead T, Bem S (eds) Emotions and beliefs: how feelings influence thoughts. Cambridge University Press, Cambridge, pp 10-44 
Damasio A (1994) Descartes' error: emotion, reason and the human brain. Papermac, London

Damasio A (1996) The somatic marker hypothesis and the possible functions of the prefrontal cortex. Philos Trans R Soc Biol Sci 351:1413-1420

Damasio A (1999) The feeling of what happens. Body and emotion in the making of consciousness. Harcourt Brace, New York

Damasio A (2003) Looking for Spinoza joy, sorrow and the feeling brain. Vintage books, London

Diwadkar VA, Flaugher B, Jones T, Zalányi L, Ujfalussy B, Keshavan MS, Érdi P (2008) Impaired associative learning in schizophrenia: behavioral and computational studies. Cogn Neurodyn 2:207-219

Dolan RJ (2002) Emotion cognition, and behavior. Science 298:1191-1194

Eich E, Kihlstrom JF, Bower GH, Forgas JP, Niedenthal PM (2000) Cognition and emotion. Oxford University Press, New York

Forgas JP (1995) Mood and judgment: the affect infusion model (AIM). Psychol Bull 11:39-66

Forgas JP (2000) Feeling is believing? The role of processing strategies in mediating affective influences on beliefs. In: Frijda $\mathrm{NH}$ et al (eds) Emotions and beliefs: how feelings influence thoughts. Cambridge University Press, Cambridge, pp 108-143

Forgas JP, Laham SM, Vargas PT (2005) Mood effects on eyewitness memory: affective influences on susceptibility to misinformation. J Exp Soc Psychol 2005:574-588

Forgas JP, Goldenberg L, Unkelbach C (2009) Can bad weather improve your memory? An unobtrusive field study of natural mood effects on real-life memory. J Exp Soc Psychol 45:254-257

Frijda NH, Manstead ASR, Bem S (eds) (2000a) Emotions and belief show feelings influence thoughts. Cambridge University Press, Cambridge

Frijda NH, Manstead ASR, Bem S (2000b) The influence of emotions on beliefs. In: Frijda NH et al (eds) Emotions and beliefs how feelings influence thoughts. Cambridge University Press, Cambridge, pp 1-9

Gazzaniga M (2009) The cognitive neurosciences. MIT Press, Cambridge

Gerstner W, Kistler WM (2002) Mathematical formulations of Hebbian learning. Biol Cybern 87:404-415

Hebb D (1949) The organisation of behavior. Wiley, New York

Jonker CM, Snoep JL, Treur J, Westerhoff HV, Wijngaards WCA (2008) BDI-modelling of complex intracellular dynamics. J Theor Biol 251:1-23

Keysers C, Gazzola V (2009) Unifying social cognition. In: Pineda JA (ed) Mirror neuron systems: the role of mirroring processes in social cognition. Humanae Press/Springer Science, Clifton, pp 3-28
Keysers C, Perrett DI (2004) Demystifying social cognition: a Hebbian perspective. Trends Cogn Sci 8:501-507

LaBar KS, Cabeza R (2006) Cognitive neuroscience of emotional memory. Nat Rev Neurosci 7:54-64

Majumdar KK (2007) A structural and a functional aspect of stable information processing by the brain. Cogn Neurodyn 1:295-303

Memon ZA, Treur J (2009) Modelling the reciprocal interaction between believing and feeling from a neurological perspective. In: Zhong $\mathrm{N}$ et al (eds) Proceedings of the first international conference on brain informatics, BI'09. Lecture Notes in Artificial Intelligence, vol 5819. Springer Verlag, New York, 2009, pp 13-24

Memon ZA, Treur J (2010) Learning to believe by feeling: an agent model for an emergent effect of feelings on beliefs. In: Zhang $\mathrm{L}$, Kwok J, Lu B-L (eds) Proceedings of the seventh international symposium on neural networks, ISNN'10, Part II. Lecture Notes in Computer Science, vol 6064. Springer Verlag, New York, 2010, pp 586-595

Niedenthal PM (2007) Embodying emotion. Science 316:1002-1005

Pessoa L (2008) On the relationship between emotion and cognition. Nat Rev Neurosci 9:148-158

Phelps EA (2006) Emotion and cognition: insights from studies of the human amygdala. Ann Rev Psychol 57:27-53

Port RF, van Gelder T (1995) Mind as motion: explorations in the dynamics of cognition. MIT Press, Cambridge, MA

Purves D, Brannon EM, Cabeza R, Huettel SA, LaBar KS, Platt ML, Woldorff MG (2008) Principles of cognitive neuroscience. Sinauer Associates Inc, Sunderland

Schooler JW, Eich E (2000) Memory for emotional events. In: Tulving E, Craik FIM (eds) The oxford handbook of memory. Oxford University Press, Oxford, pp 379-394

Seth AK (2008) Causal networks in simulated neural systems. Cogn Neurodyn 2:49-64

Spinoza B (1677/1989) Ethica. (translated by GHR Parkinson). Everyman, London

Storbeck J, Clore GL (2007) On the interdependence of cognition and emotion. Cogn Emot 21:1212-1237

Wilmer A, de Lussanet MHE, Lappe M (2010) A method for the estimation of functional brain connectivity from time-series data. Cogn Neurodyn 4:133-149

Winkielman P, Niedenthal PM, Oberman LM (2009) Embodied perspective on emotion-cognition interactions. In: Pineda JA (ed) Mirror neuron systems: the role of mirroring processes in social cognition. Humana Press/Springer Science, Clifton, pp 235-257

Xie X, Seung HS (2003) Equivalence of backpropagation and contrastive Hebbian learning in a layered network. Neural Comput 15:441-454 